\title{
催化裂解 $\mathbf{C}_{2} \mathbf{H}_{2}$ 制备空心碳球*
}

\author{
许宗祥林敬东欧延廖代伟 \\ (厦门大学化学系,物理化学研究所, 固体表面物理化学国家重点实验室, 厦门 361005)
}

\begin{abstract}
摘要 在高岭土负载的镍催化剂上, 于 $850{ }^{\circ} \mathrm{C}$ 催化裂解乙炔制备了直径约 $500 \mathrm{~nm}$ 的空心碳球. 用 XRD、TEM、 SEM 和显微激光 Raman 光谱对所得的碳球及催化剂进行了表征, 并初步探讨了反应机理 .
\end{abstract}

关键词: 空心碳球, 镍, 高岭土, 乙炔, TEM, SEM, XRD, Raman 光谱 中图分类号: $\mathrm{O} 643.35$

碳材料是与人类文明进步息息相关的重要材 料, 各种不同形态的碳单质以及碳化合物在人类发 展中做出了巨大的贡献。近年来随着富勒烯和碳 纳米管的发现, 由于这些碳族新材料具有特异的性 能, 在众多领域获得了广泛的应用, 有关的研究与 开发工作相当活跃, 越来越多的合成与表征方法为 人们所认识和使用. 最近, 一种具有笼状结构的空 心碳球 ${ }^{[1-7]}$, 由于其具有独特的功能和重要的应用价 值, 引起了人们极大的兴趣, 但多数合成方法能耗 高, 或需上千度的高温和高压, 亦或只是某些反应 的副产物, 因而产率低、分离纯化困难, 无法大量生 产, 使其应用受到限制。而催化合成法具有可以批 量生产且费用低等优点, 是一种值得进一步深人研 究的方法. 本文报导了用高岭土为载体, 镍盐为活 性组分, 乙炔气为碳源, 在氮气气氛中, 通过催化裂 解法制备空心碳球的研究结果. 我们所制备的空 心碳球, 纯度高, 无需纯化, 大小均匀, 颗粒直径在 $500 \mathrm{~nm}$ 左右, 用 TEM、SEM 和 XRD 等对空心碳球 及催化剂进行了表征, 并对该反应机理进行了初步 的探讨。

\section{1 实验部分}

\section{1 催化剂的制备}

取小于 225 目高岭土 (CP 级) 和硝酸镍 (CP 级) 以质量比 $1: 3$ 均匀混合, 加人一定量的去离子水和 $95 \%$ 乙醇, 搅拌后形成泥浆, 把泥浆涂刷于反应瓷 片上, 形成平整的小薄片, 在 $60{ }^{\circ} \mathrm{C}$ 下烘干, 即得反 应所用的催化剂前驱体。

\section{2 空心碳球的制备}

合成反应在内径 $6 \mathrm{~cm}$ 、长 $140 \mathrm{~cm}$ 的石英管中 进行, 用管式电炉加热, 电炉恒温区约 $25 \mathrm{~cm}$, 设定 升温速率为 $5{ }^{\circ} \mathrm{C} \cdot \mathrm{min}^{-1}$. 室温下将涂有催化剂前 驱体的瓷片催化剂面向下倒扣于瓷舟上, 瓷舟放置 于加热恒温区. 反应开始时先通 $\mathrm{N}_{2}$, 流速为 100 $\mathrm{mL} \cdot \mathrm{min}^{-1}$. 当温度升到 $850{ }^{\circ} \mathrm{C}$ 时, 维持恒定, 以 50 $\mathrm{mL} \cdot \mathrm{min}^{-1}$ 的流速导人 $\mathrm{C}_{2} \mathrm{H}_{2}$ 原料气。应 $2 \mathrm{~h}$ 后, 切断 $\mathrm{C}_{2} \mathrm{H}_{2}$ 源, 停止加热, 维持 $\mathrm{N}_{2}$ 气氛, 自然降温到 室温, 从瓷舟中收集的黑色粉末即为空心碳球产品.

\section{3 产品及催化剂表征}

将收集到的碳黑粉末放人肉酮溶剂中, 超声波 振荡半小时后, 滴到 TEM 专用的铜网上, 烘干, 用 JEM-100CX II型透射电子显微镜进行 TEM 测试. 反应后的催化剂片用德国产 LEO 350 型扫描电子 显微镜进行 SEM 观测. 分别取高岭土载体粉末, 未反应和反应后的催化剂研磨所得的粉末, 以及反 应后收集所得的产品, 在 $\mathrm{D} / \mathrm{max}-\mathrm{rC}$ 型转靶 $\mathrm{X}$ 射线 衍射仪上进行 $X$ 射线粉末衍射实验, 衍射条件为 $\mathrm{Cu} K_{\alpha}, 40 \mathrm{kV}, 30 \mathrm{~mA}$, 扫描速率 $4^{\circ} \cdot \mathrm{min}^{-1}$. 反应所 得样品的拉曼光谱是在室温下用 Labram I. Dilor 型 显微激光拉曼光谱仪测量, 激发光源为 $632.8 \mathrm{~nm}$ 的 $\mathrm{He}-\mathrm{Ne}$ 激光线, 激光到达样品表面的功率为 2.5 $\mathrm{mW}$.

\section{2 结果与讨论}

\section{1 产品表征结果与讨论}

由样品 TEM 表征所得的图 1(a)可以看出在放

2003-04-25 收到初稿, 2003-07-07 收到修改稿。 联系人: 廖代伟(E-mail: dwliao@ xmu. edu. cn; Tel: 0592-2183045;

Fax: 0592-2183043)。*国家自然科学基金（20273053、20023001、29933040)资助项目 

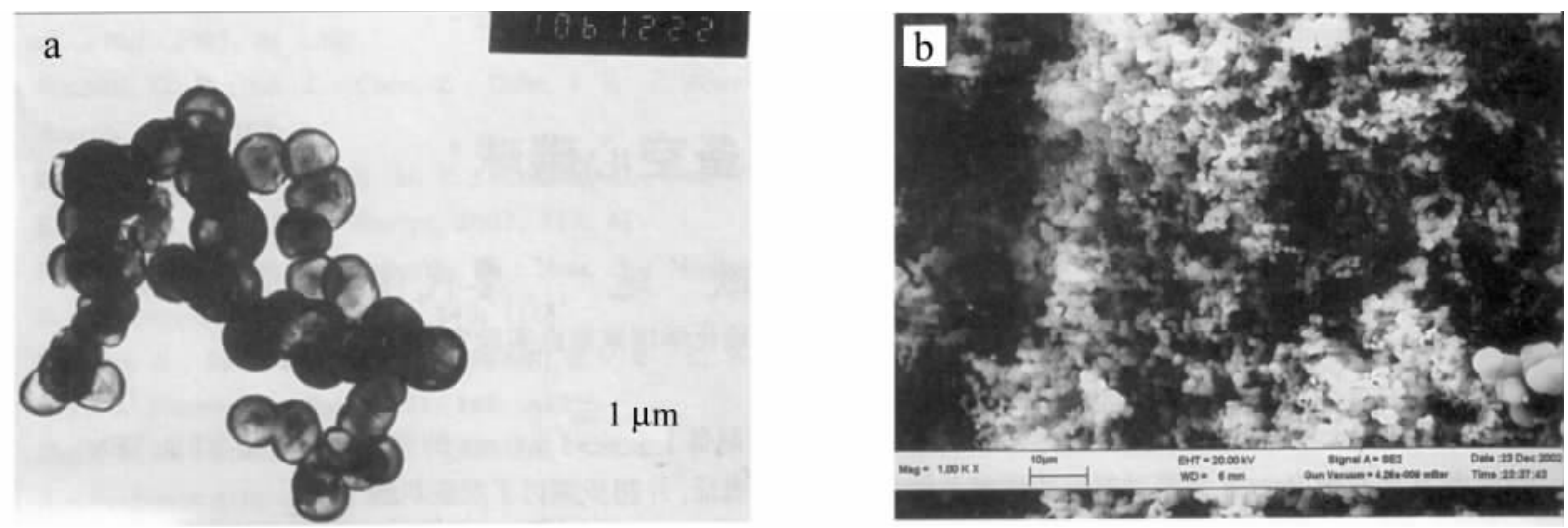

图 1 空心碳球的 $\operatorname{TEM}(\mathrm{a})$ 和 $\operatorname{SEM}(\mathrm{b})$ 表征图

Fig. 1 TEM image (a) and SEM image (b) of the hollow carbon spheres

大 1 万倍下, 收集所得的样品 TEM 形貌与文献 ${ }^{[5]}$ 报 导的空心碳球一样, 为中空的规则圆形颗粒, 大小比 较均一, 在 $500 \sim 600 \mathrm{~nm}$ 左右. 产品不需纯化, 具有 高纯的特点, 单程产量可达 $0.2 \mathrm{~g}$. 从 SEM 表征图 1 (b) 可以看出所制备的碳球不象一般的催化裂解法 制备的碳纳米管那样会含有催化剂和不定型碳等其 它杂质.

图 2 和图 3 分别为空心碳球的 XRD 图和 Raman 光谱图.从碳球的 XRD 谱图 (图 2)可以看出所制 备的碳球纯度高, 基本无杂质峰, 图中的两个峰都是 碳的特征峰, $2 \theta=25.16^{\circ}$ 和 $43.76^{\circ}$ 分别归属于 C (002) 和 C (100), 是石墨峰. 从图 3 可看出所制备 的空心碳球与一般的多壁碳纳米管 (MWCNTs) 具 有类似的微观结构, 都有跟石墨片层相似的结构, 因 此可观测到相似的拉曼光谱. 但由于在结构和尺寸 等方面的差异, 二者的谱图仍有所不同。本实验制 备的碳球拉曼光谱, 其两个 $E_{2 \mathrm{~g}}$ 拉曼活性振动模式 产生的强峰出现在 $1581.39 \mathrm{~cm}^{-1}$, 归属于碳球中的 石墨化碳, 指认为 $\mathrm{G}$ 模, 与文献 ${ }^{[1]}$ 报导的多壁碳纳 米管拉曼光谱的 $1580 \mathrm{~cm}^{-1}$ 峰基本一致 . 其另一

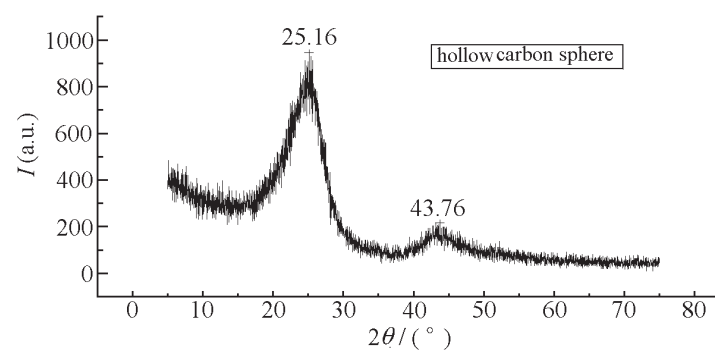

图 2 空心碳球的 XRD 图

Fig. 2 XRD pattern of the hollow carbon spheres
个强峰则出现在 $1319.34 \mathrm{~cm}^{-1}$, 归属于碳球中的非 石墨化碳, 主要是结构缺陷引起的, 指认为 $\mathrm{D}$ 模, 则 与 MWCNTs 的 $1350 \mathrm{~cm}^{-1}$ 峰 $[1]$ 有较大区别. 这可 能是由于碳球中的石墨晶格结构不完整度与 MWCNTs 不同, 使其产生的振动峰有所区别。同时 从光谱图中可以看出碳球的 $\mathrm{D}$ 模强度比 $\mathrm{G}$ 模强度 大, 说明所得的碳球中无序结构的碳较多.

\section{2 催化剂表征结果与讨论}

图 4 是未负载镍的高岭土, 以及负载镍后的高 岭土反应前后的 XRD 谱图. 图中曲线 (1) 对应于 未负载镍的高岭土, 其中 $2 \theta=12.38^{\circ}(001) 、 24.92^{\circ}$ (002)、 $37.76^{\circ}(202)$ 和 $35.02^{\circ}(130)$ 等谱峰对应的是 高岭土中的高岭石; $2 \theta=8.86^{\circ}$ 和 $17.8^{\circ}$ 对应的是水 云母; $2 \theta=26.6^{\circ}$ 对应的是石英; $20.38^{\circ}$ 附近可能是 高岭石和石英谱峰的混合. 从曲线 (2)可以看出, 当 高岭土负载镍盐后, 其 XRD 图有明显的变化, 多了 几个峰, 其中 $2 \theta=10.9^{\circ} 、 13.72^{\circ}$ 和 $16.68^{\circ}$ 均应归属 于 $\mathrm{Ni}$ 峰. 而反应后的催化剂, 通过 XRD 检测得到 的曲线 (3), 其中两个峰 $2 \theta=26.56^{\circ}$ 和 $2 \theta=44.34^{\circ}$ 最强, 应该分别归属于碳 (002) 和 (100). 该结果与

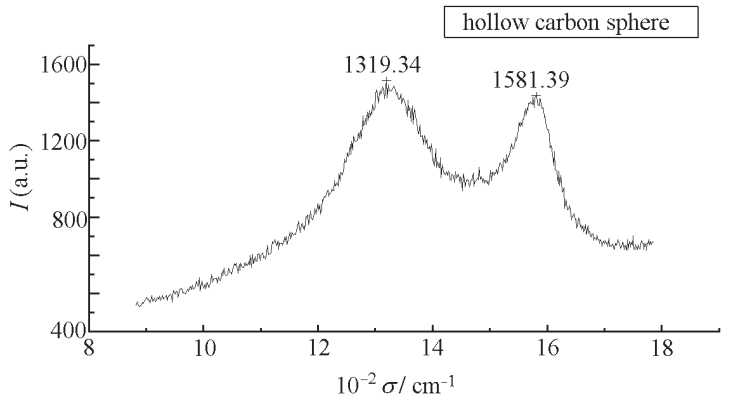

图 3 空心碳球的 Raman 光谱图

Fig. 3 Raman spectrum of the hollow carbon sphere 


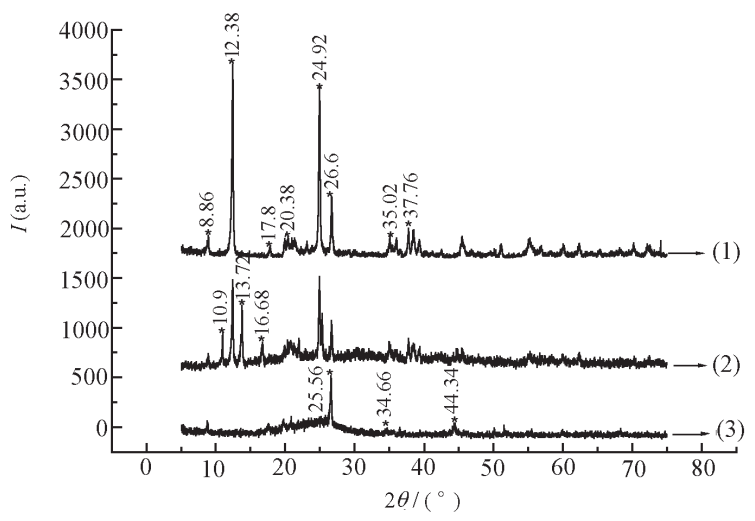

图 4 反应前后的催化剂及高岭土载体的 XRD 图

Fig. 4 XRD patterns for the pure kaolin and catalysts

(1) pure kaolin; (2)catalyst before reaction;

(3) catalyst after reaction

反应后催化剂片的 SEM 图 (图 5)一致,反应后的催 化剂片为碳球层所包覆. 同时由 XRD 谱峰对比可 以看出, 经过高温反应后, 高岭石对应谱峰基本都消 失, 高岭土中的高岭石结构受到彻底的破坏, 而石英 相和水云母则能稳定地存在下来, 受破坏的高岭石 转变为非晶态物质. 从 XRD 图中也可以看出反应 后催化剂中还含有少量的方石英 $\left(2 \theta=34.66^{\circ}\right)$, 可 能是高岭土经过高温反应后局部高温的产物. 我们 认为, 高岭土在高温反应中的这种结构变化, 对于镍 催化剂催化裂解 $\mathrm{C}_{2} \mathrm{H}_{2}$ 形成碳球具有促进作用.

由于高岭土的特性, 采用高岭土为载体的催化 剂反应后呈成片状, 完整地保留了有关的反应信息. 取反应后的催化剂片进行扫描电镜检测, 从图 5 中 可以看出, 残留在催化剂片上的碳球大小远小于我 们收集所得的碳球, 在 $100 \mathrm{~nm}$ 左右. 说明反应中同 时形成了不同大小级别的碳球, 但在 $\mathrm{N}_{2}$ 气的吹扫作 用下, 较大颗粒的碳球, 脱离催化剂表面掉到反应瓷 舟中, 而小颗粒的碳球或残留在催化剂片上, 或随 $\mathrm{N}_{2}$ 气吹走.

化学气相沉积法 (CVD) 制备碳纳米管 (CNTs) 的生长机理可能是碳源气在催化剂金属颗粒的特定 晶面上吸附、分解而生成碳物种 (碳原子), 然后碳物 种溶人金属颗粒体相, 经扩散迁移至金属颗粒背面, 进而形成 CNTs ${ }^{[8]}$. 本反应与一般的 CVD 法制备 CNTs 过程类似, 只是催化剂的载体不同. 在反应 的高温下, 镍盐被 $\mathrm{C}_{2} \mathrm{H}_{2}$ 气体还原形成金属颗粒, 这 些金属颗粒负载于高岭土上大小不同的孔结构中. 随着反应的进行, $\mathrm{C}_{2} \mathrm{H}_{2}$ 在这些金属颗粒上裂解, 形 成碳物种. 由于高岭土在高温下的某些相变作用,

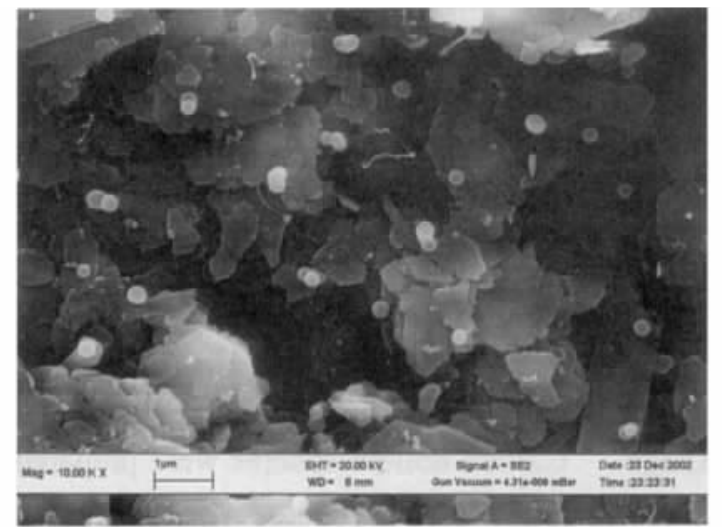

图 5 反应后催化剂片的 SEM 表征图

Fig. 5 SEM image of the catalgst after reaction

使形成的碳物种在这些孔上形成大小不同的碳球. 然后在 $\mathrm{N}_{2}$ 气吹扫下脱离金属颗粒. 所以, 未在碳球 中发现催化裂解法制备碳纳米管时都会产生的金属 颗粒和不定型碳等其它杂质, XRD 谱峰也未显示碳 球中有其它杂质. 更详细的反应机理有待于进一步 的研究.

\section{References}

1 Cheng, H. M. Carbon nanotubes synthesis, microstructure, properties and applications. Beijing: Chemical Industry Press, 2002: 152 [成会明. 纳米碳管制备, 结构, 物性及应用. 北 京: 化学工业出版社, 2002: 152]

2 Niwase, K. ; Homae, T. ; Nakamura, G. K. ; Kondo, K. Chem. Phys. Lett., 2002, 36 (2): 47

3 Xu, B. S. ; Tanaka, A. Acta Mater., 1998, 46 (15): 5249

4 Wurmser, G. ; Konnoa, H. ; Kaburagib, Y. ; Oshida, K. ; Inagaki, M. Synthetic Metal., 2001, 11(8): 33

5 Wang, Z. L. ; Yin, J. S. Chem. Phy. Lett., 1998, 28 (9): 189

6 Stockli, T. ; Bonard, J. M. ; Chatelain, A. Phys. Rev. B, 2000, 61 (8): 571

7 Terones, H. ; Terones, M. J. Phys. Chem. Solids, 1997, 58 (11): 1789

8 Zhang, H. B. ; Lin, G. D. ; Tsai, K. R. J. Xiamen Univ. (Nat. Sci. ), 2001, 40 (2): 387 [张鸿斌, 林国栋, 蔡启瑞. 厦门大学学报 (Xiamen Daxue Xuebao), 2001, 40 (2): 387]

9 Wu, G. T. ; Zhu, G. M. ; You, J. K. ; Lin, Z. G. Chem. J. Chinese Universities, 2002, 23 (1): 98 [吴国涛, 朱光明, 尤金跨, 林祖 庚. 高等学校化学学报 (Gaodeng Xиexiao Huaxue Xиebao), 2002, 23 (1): 98]

10 Chen, P. ; Wang, P. F. ; Lin, G. D, ; Zhang, H. B. Chem. J. Chinese Universities, 1995, 16(11): 1783 [陈 萍, 王陪峰, 林国栋, 张鸿斌. 高等学校化学学报 (Gaodeng Xuexiao Huaxue Хиеbao), 1995, 16 (11): 1783 ] 


\section{Preparation of Hollow Carbon Spheres by Catalytic Pyrolysis of $\mathrm{C}_{2} \mathrm{H}_{2}{ }^{*}$}

Xu Zong-Xiang Lin Jing-Dong Ou Yan Dai-Wei

( State Key Laboratory for Physical Chemistry of the Solid Surface, Department of Chemistry, Institute of Physical Chemistry,

Xiamen University, Xiamen 361005 )

Abstract Carbon hollow spheres were prepared via catalytic pyrolysis with kaolin supported nickel nitrate as catalyst. The hollow carbon spheres were synthesized by the catalytic pyrolysis of $\mathrm{C}_{2} \mathrm{H}_{2}$ mixed with $\mathrm{N}_{2}$ at $850{ }^{\circ} \mathrm{C}$. The characterization of catalyst and hollow carbon spheres were carried out by XRD, TEM, SEM and Raman spectroscopy. The TEM and SEM images suggested that the carbon spheres with diameter about $500 \mathrm{~nm}$ were hollow. SEM images and XRD patterns of catalyst suggested some information about the possible formation mechanism of hollow spheres.

Keywords: Hollow carbon sphere, Nickel, Kaolin, $\mathrm{C}_{2} \mathrm{H}_{2}$, TEM, SEM, XRD, Raman spectroscopy

Received: April 25, 2003; Revised: July 7, 2003. Correspondent: Liao Dai-Wei (E-mail: dwliao@ xmu. edu. cn; Tel: 0592-2183045; Fax: 0592-2183043). $\quad$ *The Project Supported by NSFC (20273053, 20023001, 29933040) 